\title{
Article \\ Gas Porosimetry by Gas Adsorption as an Efficient Tool for the Assessment of the Shaping Effect in Commercial Zeolites
}

\author{
Alejandro Orsikowsky-Sanchez ${ }^{1,2, *}$, Christine Franke ${ }^{3}$, Alexander Sachse ${ }^{4}\left(\mathbb{D}\right.$, Eric Ferrage ${ }^{4} \mathbb{(}$, Sabine Petit ${ }^{4}(\mathbb{D}$, \\ Julien Brunet ${ }^{4}$, Frédéric Plantier ${ }^{2}$ and Christelle Miqueu ${ }^{2, *(1)}$
}

1 TOTAL EP_Pôle d'Etudes et de Recherche de Lacq (PERL), BP 64170 Lacq, France

2 Laboratoire des Fluides Complexes et leurs Réservoirs, Université de Pau et des Pays de l'Adour, E2S UPPA, CNRS, 64600 Anglet, France; frederic.plantier@univ-pau.fr

3 MINES ParisTech, Center of Geosciences, PSL University, CEDEX, 77305 Fontainebleau, France;

christine.franke@mines-paristech.fr

4 Université de Poitiers-IC2MP, UMR 7285 CNRS, 86073 Poitiers, France; alexander.sachse@univ-poitiers.fr (A.S.); eric.ferrage@univ-poitiers.fr (E.F.); sabine.petit@univ-poitiers.fr (S.P.); julien.brunet@univ-poitiers.fr (J.B.)

* Correspondence: alejandro.orsikowsky@total.com (A.O.-S.); christelle.miqueu@univ-pau.fr (C.M.)

\section{check for} updates

Citation: Orsikowsky-Sanchez, A.; Franke, C.; Sachse, A.; Ferrage, E.; Petit, S.; Brunet, J.; Plantier, F.; Miqueu, C. Gas Porosimetry by Gas Adsorption as an Efficient Tool for the Assessment of the Shaping Effect in Commercial Zeolites. Nanomaterials 2021, 11, 1205. https://doi.org/ 10.3390/nano11051205

Academic Editor:

Diego Cazorla-Amorós

Received: 27 March 2021

Accepted: 23 April 2021

Published: 1 May 2021

Publisher's Note: MDPI stays neutral with regard to jurisdictional claims in published maps and institutional affiliations.

Copyright: (c) 2021 by the authors. Licensee MDPI, Basel, Switzerland. This article is an open access article distributed under the terms and conditions of the Creative Commons Attribution (CC BY) license (https:/ / creativecommons.org/licenses/by/ $4.0 /)$.

\begin{abstract}
A set of three commercial zeolites (13X,5A, and 4A) of two distinct shapes have been characterized: (i) pure zeolite powders and (ii) extruded spherical beads composed of pure zeolite powders and an unknown amount of binder used during their preparation process. The coupling of gas porosimetry experiments using argon at $87 \mathrm{~K}$ and $\mathrm{CO}_{2}$ at $273 \mathrm{~K}$ allowed determining both the amount of the binder and its effect on adsorption properties. It was evidenced that the beads contain approximately $25 \mathrm{wt} \%$ of binder. Moreover, from $\mathrm{CO}_{2}$ adsorption experiments at $273 \mathrm{~K}$, it could be inferred that the binder present in both 13X and 5A zeolites does not interact with the probe molecule. However, for the $4 \mathrm{~A}$ zeolite, pore filling pressures were shifted and strong interaction with $\mathrm{CO}_{2}$ was observed leading to irreversible adsorption of the probe. These results have been compared to XRD, IR spectroscopy, and ICP-AES analysis. The effect of the binder in shaped zeolite bodies can thus have a crucial impact on applications in adsorption and catalysis.
\end{abstract}

Keywords: zeolite; binder; $\mathrm{CO}_{2}$; gas porosimetry; adsorption

\section{Introduction}

Zeolites are porous materials that have been widely used in industrial applications of adsorption (gas or liquid) and catalysis for more than 60 years [1]. These materials have the major advantage of superior thermal and mechanical stability. Moreover, zeolites are the adsorbents of choice related to their low cost. To study the adsorption mechanisms, adsorbents must be as pure as possible. Thus, adsorbents in powder form (absence of binder) are most suitable. However, in dynamic pilot experiments and industrial processes shaped adsorbents are used to limit the pressure drop. These shaped adsorbents are typically obtained by the extrusion of pure powders through the employment of a binder that provides mechanical strength and cohesion of the individual crystals [1-5]. The most common binders used to shape zeolites are clays (kaolin, metakaolin, attapulgite, bentonite), other inorganic compounds $\left(\mathrm{SiO}_{2}, \mathrm{Al}_{2} \mathrm{O}_{3}\right)$, and some organic compounds (carboxymethylcellulose, methylcellulose, lignosulfonate) [1,4]. Yet, in most studies, the impact of the binder on the adsorption mechanisms is scarcely described. Within this limited information, most of the reports focus on the impact of binders in catalysis and in the mechanical properties rather than on adsorption behavior [2,6]. Indeed, Gilson and co-workers very recently observed that binders can react and thus importantly impact acidic properties of zeolites [7]. Some other authors analyzed the effect of the amount of binder on zeolite pore volume [5]. For instance, among the studies that have analyzed the binder effect on gas adsorption, 
Charkhi et al. [8] investigated the influence of bentonite on the adsorption properties of a granulated nano NaY zeolite through Xenon and Nitrogen adsorption. They showed that increasing the bentonite content from 20 to $40 \mathrm{wt} \%$ favors the rupture of load granules by $232 \%$ and decreases the BET surface area by $66 \%$. Further, $25 \mathrm{wt} \%$ of binder caused a decrease in the Xenon crystal diffusivity. Jasra et al. [9] analyzed the effect of clay binders on the sorption and catalytic properties of mordenite and HY pellets by using the adsorption of $\mathrm{N}_{2}, \mathrm{O}_{2}$, Ar and $\mathrm{CH}_{4}$, and X-ray diffraction (XRD). Their study suggests an increase in the surface heterogeneity of zeolites upon pelletization due to the migration of clay cations inside the zeolite cavities. Sun et al. [10] investigated the effect of binders on the adsorption of $\mathrm{N}$-paraffins in 5A zeolite by comparing the amount adsorbed on the pure zeolite and the shaped one through electron microscopy. They concluded that the 5A zeolite with binder shows lower adsorption capacities due to the dilution effect as well as the blocking of pore apertures. More recently, Chen et al. [11] studied the impact of the interactions between a silica binder and a NaY zeolite using XRD, Transmission Electron Microscopy (TEM), and Fourier Transform Infrared (FTIR) spectroscopy techniques, regarding both the structure and the surface effects. They observed that the interactions between the zeolite and the binder, combined with the dealumination of the framework, leads to a decrease in crystal size and crystallinity, and an increase of $\mathrm{Si} / \mathrm{Al}$ ratio, hydrophobicity, and thermal stability. IR spectra of $\mathrm{NH}_{3}$ adsorption on $\mathrm{NaY}$ and $\mathrm{NaY}-\mathrm{SiO}_{2}$ revealed that pelletization caused an increase in the number of Brønsted acid sites. Besides, Cao et al. [12] evaluated the impact of the alumina binder by measuring heats of adsorption of $\mathrm{SF}_{6}$ and $\mathrm{CO}_{2}$ on silicalite-1 pellets via calorimetric measurements. Their results indicate that the strong adsorption of $\mathrm{CO}_{2}$ on alumina binders leads to a significant heterogeneity on the pelletized silicalite-1 sample. Finally, Shams et al. [13] reported the effect of binders composed of kaolin and carboxymethylcellulose (CMC) on the sieving/adsorption properties of a 5A monolith. They characterized the sample using XRD, Scanning Electron Microscopy (SEM), and Energy-dispersive X-ray (EDX) spectroscopy and concluded that the highest ion exchange capacity and the best sieving results were obtained with $30 \mathrm{wt} \%$ of binders (kaolin), and that the use of small amounts of CMC has profound effects on the adsorption properties of 5 A zeolite.

It becomes clear from these studies that the effect of the binders is not neglectable, in particular if the objective is to study the adsorption mechanisms on shaped samples. The present work aims to propose an efficient control protocol based on gas porosimetry to evaluate the effect of the binder. The comparison of the adsorption of an inert probe molecule such as argon and a molecule with a smaller kinetic diameter and a quadrupole moment such as $\mathrm{CO}_{2}$ on both powdered and shaped samples are used for this purpose. The results are compared to XRD, IR spectroscopy, and Induced Coupled Plasma-Atom Emission Spectroscopy (ICP-AES) analysis, not to identify the binder but rather to assess the protocol.

\section{Materials and Methods}

\subsection{Materials}

Three commercial zeolites were considered in this study under two different shapes: pure zeolite powder and spherical beads composed of zeolite powder and a binder of unknown composition and quantity. All zeolite samples were provided by Fisher Scientific (Waltham, MA, USA).

For both 13X and 5A zeolites, two different samples of spherical beads of distinct origin (i.e., two different providers) were compared with one sample of zeolite powder (Table 1). It should be noted that the powder and SB1 samples come from the same provider. In the case of the $4 \mathrm{~A}$ zeolite, only one shaped sample was available for comparison with the powder sample. The nomenclature adopted in this work is as shown in Table 1. 
Table 1. Zeolite samples studied in this work.

\begin{tabular}{cccc}
\hline Zeolite Sample & 13X & 5A & 4A \\
\hline Powder (P) & Z13X_P & Z5A_P & Z4A_P \\
Spherical beads 1 (SB1) & Z13X_SB1 & Z5A_SB1 & Z4A_SB1 \\
Spherical beads 2 (SB2) & Z13X_SB2 & Z5A_SB2 & - \\
\hline
\end{tabular}

Argon, helium, and carbon dioxide for gas adsorption experiments were provided by Linde Gas. The first two gases were purchased in 6.0 quality (purity $\geq 99.9999 \%$ ) and the $\mathrm{CO}_{2}$ had a quality of 4.5 (purity $\geq 99.995 \%$ ).

\subsection{Method}

\subsubsection{Gas Porosimetry}

All adsorption isotherms were measured using a commercial volumetric apparatus. Argon adsorption isotherms at $87 \mathrm{~K}$ were performed using an Autosorb-iQ (Quantachrome Instruments, Anton Paar, Graz, Austria) with the Cryosync ${ }^{\circledR}$ temperature regulation system. Carbon dioxide adsorption isotherms at $273 \mathrm{~K}$ were carried out employing an ASAP2020 (Micromeritics Instruments Corporation, Norcross, GA, USA) and an Autosorb-iQ (Quantachrome Instruments, Anton Paar, Graz, Austria). Temperature regulation at $273 \mathrm{~K}$ was implemented with an ice-water mixture Dewar and with a double jacket Dewar and a thermostatic bath respectively. To eliminate any trace of gas prior to the experiment, all the samples were degassed during $12 \mathrm{~h}$ at $573 \mathrm{~K}$ under secondary vacuum (heating ramp of $10 \mathrm{~K} \mathrm{~min}^{-1}$ ). For further details on these conditions of pretreatment please see Refs [14,15].

\subsubsection{X-ray Diffraction Analysis}

The XRD patterns were collected using a PANalyticalX'Pert Powder X-ray diffractometer (Malvern Panalytical, Malvern, UK) equipped with a Cu-radiation and an X'celerator detector. The analyses were conducted on homogenized dry bulk samples prepared on randomly oriented powder mounts and were scanned in the 2 to $60^{\circ} 2 \Theta$ angular range. The XRD patterns have been interpreted using the EVA@ software (Bruker DIFFRAC Plus 2007, version V5; for more details see Giencke [16]). Reference files from the International Centre for Diffraction Data (ICDD PDF2 data files) have been used for mineral phase identification. (Semi) quantification of the present mineral phases was made using the normalized intensity ratio (RIR) method with an uncertainty of $\pm 5 \%$ (e.g., Chung et al. [17]).

\subsubsection{Infrared Spectroscopy}

Fourier transform infrared (FTIR) spectra were measured in transmission mode at $4 \mathrm{~cm}^{-1}$ resolution, in the $4000-400 \mathrm{~cm}^{-1}$ range using a Magna-IR 7600 Nicolet spectrometer (ThermoFisher Scientific, Waltham, MA, USA) equipped with an EverGlo source, a $\mathrm{KBr}$ beam splitter, and a DTGS-KBr detector. Spectra were measured from $\mathrm{KBr}$ pressed pellets dried overnight at $110{ }^{\circ} \mathrm{C}$ before measurement to remove adsorbed water. The pellets were prepared by mixing $100 \mathrm{mg}$ of $\mathrm{KBr}$ and $0.5 \mathrm{mg}$ (low sample concentration) and $1 \mathrm{mg}$ powdered sample (high sample concentration). The low sample concentration allows avoiding the oversaturation in the strong silicate absorption regions in the $1000-1100 \mathrm{~cm}^{-1}$ and $400-550 \mathrm{~cm}^{-1}$ regions, while the high sample concentration allows for higher resolution of the small bands. The spectral subtractions were made with the Omnic software (ThermoFisher Scientific, MA, Waltham, USA). FTIR spectra were also measured in Diffuse Reflection Infrared Spectroscopy (DRIFTS) mode with an Agilent 4100 ExoScan spectrometer (Santa Clara, CA, USA). This FTIR spectrometer equipped with an external reflectance probe allows measuring diffuse reflectance spectra of powders as well as of spherical beads without any specific preparation. In DRIFTS, when coming in to contact with the sample, the infrared light passes through the top surface of the sample before being reflected back out of the sample and into the detector of the spectrometer. Even if distortions from specular reflectance artifacts cannot be excluded, qualitative spectral 
comparisons could be done. The background was performed on a silicium wafer reference, the acquisition time was $30 \mathrm{~s}$ and the resolution was $4 \mathrm{~cm}^{-1}$.

\subsubsection{ICP-AES Analysis}

The chemical nature of the $4 \mathrm{~A}$ samples was determined through Induced Coupled Plasma-Atom Emission Spectroscopy (ICP-AES) on a Perkin Elmer Optima 2000 DV apparatus (Waltham, MA, USA).

\section{Results and Discussion}

\subsection{Gas Porosimetry}

\subsubsection{Argon Adsorption-Desorption at $87 \mathrm{~K}$}

Figure 1 compares the argon adsorption-desorption (fully reversible) isotherms at $87 \mathrm{~K}$ on the $13 \mathrm{X}$ and $5 \mathrm{~A}$ zeolite samples throughout the entire relative pressure range and shows that the isotherms are fully reversible.

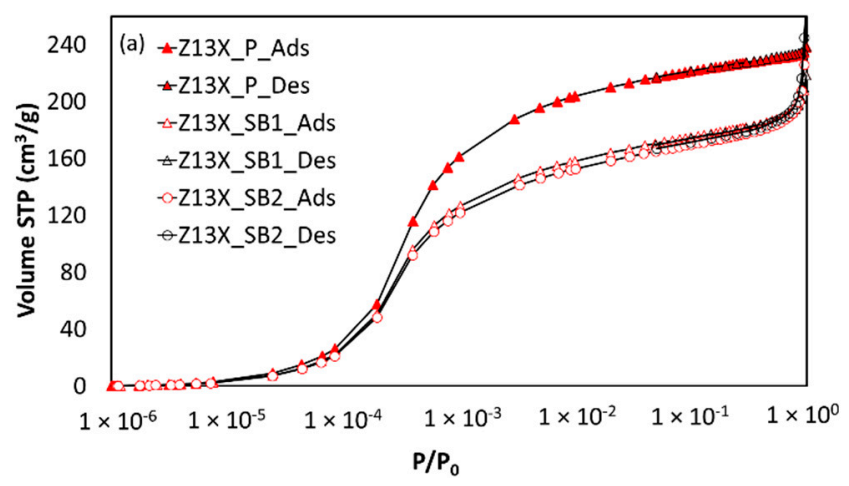

(a)

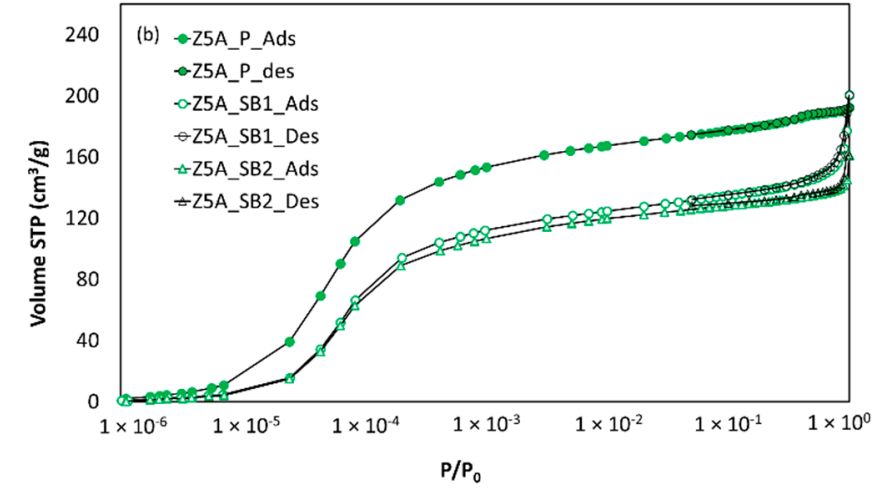

(b)

Figure 1. Argon adsorption-desorption isotherms at $87 \mathrm{~K}$ on different zeolites samples. (a) 13X zeolite, and (b) 5A zeolite.

From Figure 2, it can be inferred that the $4 \mathrm{~A}$ zeolite sample does not allow for the uptake of argon in its micropores, as significant diffusional limitations and molecular crowding phenomena are encountered for pore sizes lower than $4.5 \AA$ for argon at $87 \mathrm{~K}[14,18]$.

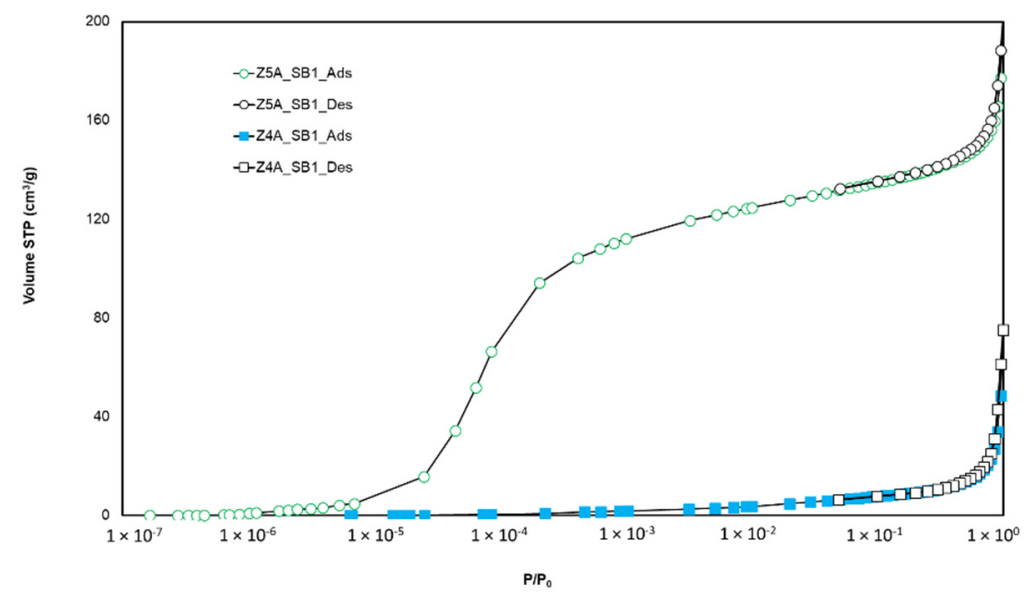

Figure 2. Comparison of argon adsorption-desorption isotherms at $87 \mathrm{~K}$ on $5 \mathrm{~A}$ and $4 \mathrm{~A}$ zeolites in spherical bed shape.

The Pore Size Distribution (PSD) and the micropore volume of each sample were obtained by applying the NLDFT model for argon adsorption on cylindrical/spherical pores of zeolites available in the Quantachrome's ASiQwin Software. The BET surface 
area was calculated according to the procedure defined for microporous adsorbents by

Rouquerol et al. [19]. These results are shown in Figure 3 and Table 2, respectively.

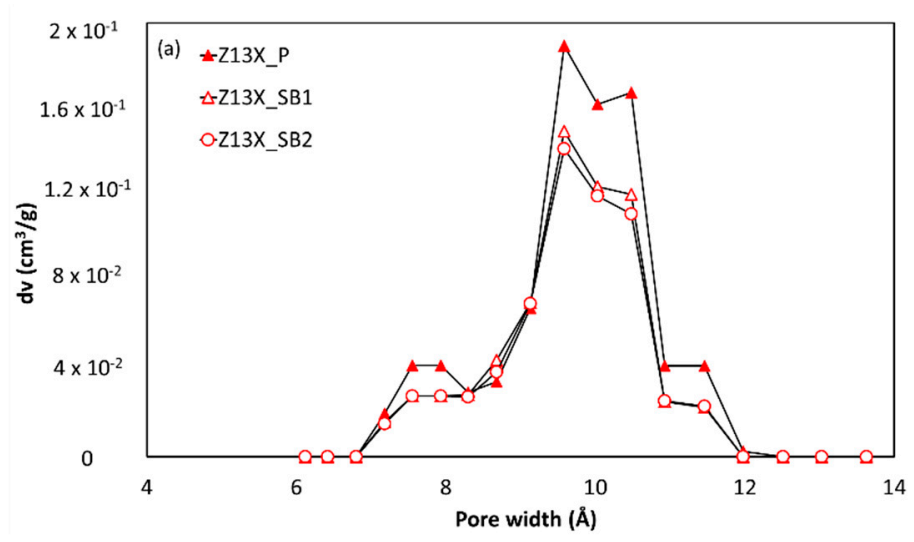

(a)

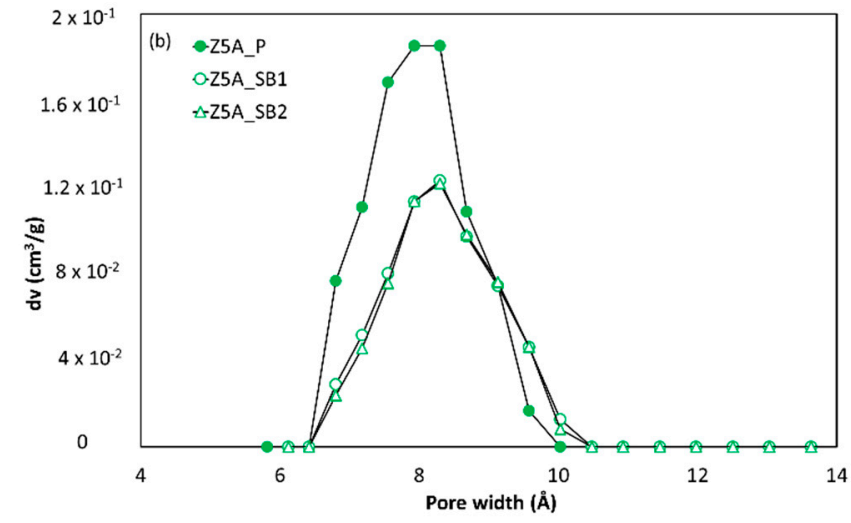

(b)

Figure 3. Pore size distribution of different zeolite samples. (a) 13X zeolites, and (b) 5A zeolites obtained with a NLDFT model (Quantachrome's ASiQwin Software).

Table 2. BET surface area and micropore volumes of different zeolite samples.

\begin{tabular}{ccccccc}
\hline \multirow{2}{*}{ Zeolite Sample } & \multicolumn{3}{c}{ 13X } & \multicolumn{3}{c}{ 5A } \\
\cline { 2 - 7 } & Z13X_P & Z13X_SB1 & Z13X_SB2 & Z5A_P & Z5A_SB1 & Z5A_SB2 \\
\hline S $_{\text {BET }}\left(\mathrm{m}^{2} / \mathrm{g}\right)$ & 799 & 624 & 607 & 641 & 482 & 461 \\
$\mathrm{~V}_{\mathrm{p}}(\mathrm{NLDFT})$ & 0.37 & 0.28 & 0.28 & 0.35 & 0.25 & 0.23 \\
$\left(\mathrm{~cm}^{3} / \mathrm{g}\right)$ & & & & & & \\
\hline
\end{tabular}

The adsorbed amount, the micropore volume, and the BET surface area have been measured on dry samples. The observed difference in Figure 1 and Table 2 between powder and spherical beads samples can be attributable to the presence of the binders contained in the shaped samples. The difference between powders and beads is the same for BET surface area as for the pore volume, which proves that the binder does not influence the porosity of the sample (pore blocking). The amount of binder may vary depending on the adsorbent manufacturer and can be calculated from the difference in adsorbate amounts, pore volumes, and BET surfaces between powder and shaped simples. Hence, from the differences between both the pore volumes and surfaces between the pure zeolites (powders) and the pelletized samples given in Table 2, the binder amount can be estimated between $22-24 \mathrm{wt} \%$ of the beads for $13 \mathrm{X}$ zeolites and to $24-28 \mathrm{wt} \%$ in the case of $5 \mathrm{~A}$ zeolites, respectively. These results are in very good agreement with the literature [1,2,5].

The PSDs in Figure 3 are identical for both powder and spherical beads forms. Although 13X zeolite is composed of $7.4 \AA$ windows and $13 \AA$ cages [20], a single pore-filling mechanism is observed in the argon adsorption isotherm at $87 \mathrm{~K}$ (relative pressure interval between $10^{-5}$ and $10^{-3}$ approximately). That explains the obtaining of an "average" pore size of around $10 \AA$ observed in the PSD. Likewise, $5 \mathrm{~A}$ zeolite is composed of $5 \AA$ windows and $11 \AA$ cages [20] and the observed mean pore size in the PSD is around $8 \AA$. Besides, for spherical beads PSDs are similar, indicating almost the same micropore volume per unit of "porous" mass. According to the argon gas porosimetry results at $87 \mathrm{~K}$, even if the spherical beads samples come from different providers, it is possible to state that the binder does not modify the adsorption properties of pure zeolites but logically impacts the surface area and micropore volume available to adsorption per unit mass of the sample. Finally, the more important argon uptake observed in the high relative pressure range $(\mathrm{P} / \mathrm{P} 0 \rightarrow 1)$ indicates that the extrusion process leads to some degree of inter-particular meso- and/or macro-porosity. 


\subsection{2. $\mathrm{CO}_{2}$ Adsorption-Desorption at $273 \mathrm{~K}$}

Figure 4 shows the $\mathrm{CO}_{2}$ adsorption-desorption isotherms at $273 \mathrm{~K}$ obtained for all the zeolite samples considered in this work. All the measurements were performed up to $101.3 \mathrm{kPa}$ (i.e., $\mathrm{P} / \mathrm{P}_{0}=3.10^{-2}$ ).

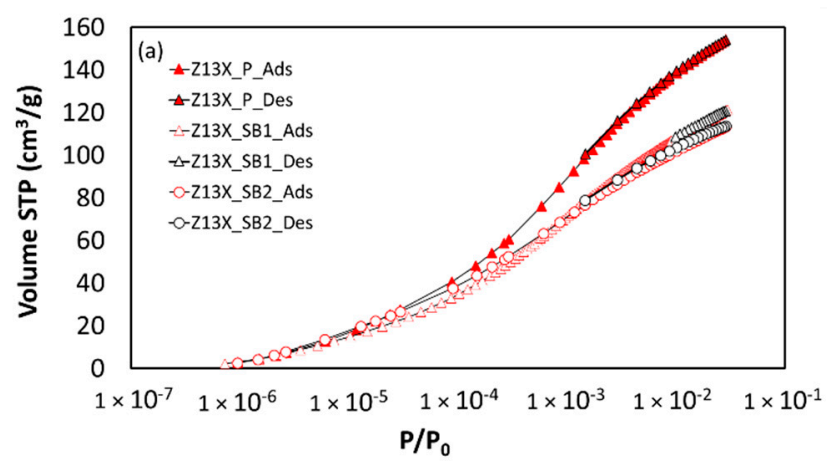

(a)

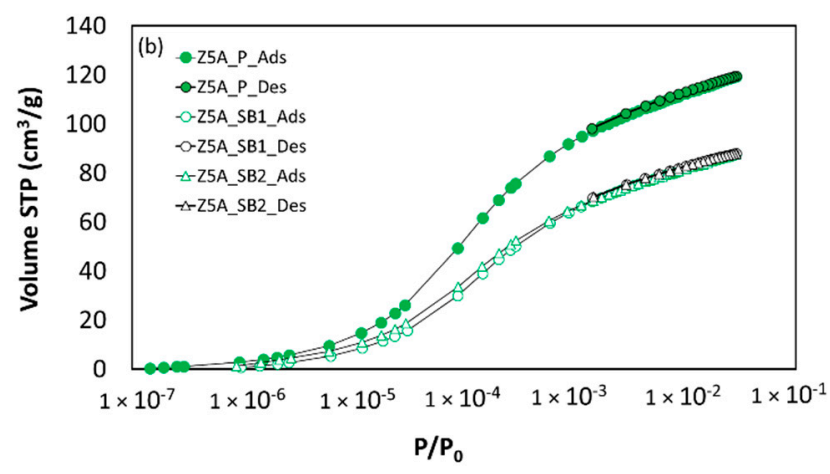

(b)

Figure 4. $\mathrm{CO}_{2}$ adsorption-desorption isotherms at $273 \mathrm{~K}$ on different zeolite samples: (a) $13 \mathrm{X}$ zeolites, and (b) $5 \mathrm{~A}$ zeolites.

$\mathrm{CO}_{2}$ isotherms at $273 \mathrm{~K}$ allow evaluating if the difference observed on powder and spherical beads from argon isotherms at $87 \mathrm{~K}$ is recovered or if the binder has an impact on the $\mathrm{CO}_{2}$ adsorption. In addition, in the case of some materials such as $4 \mathrm{~A}$ zeolite in this work, $\mathrm{CO}_{2}$ adsorption is the only possibility to evaluate some structural key information using the gas porosimetry technique, as it is the only probe molecule able to enter the ultramicropores [18].

Regarding $\mathrm{CO}_{2}$ adsorption isotherms at $273 \mathrm{~K}$ for $13 \mathrm{X}$ and $5 \mathrm{~A}$ zeolites (Figure 4), the difference in adsorbed volume between powder and granular beads samples is between 22 and $24 \mathrm{wt} \%$ in the case of $13 \mathrm{X}$ zeolite samples and between 24 and $28 \mathrm{wt} \%$ for $5 \mathrm{~A}$ zeolite samples, respectively. Even if these zeolites contain different cations $\left(\mathrm{Ca}^{2+}, \mathrm{Na}^{+}\right.$for $5 \mathrm{~A}$ zeolite and $\mathrm{Na}^{+}$for $13 \mathrm{X}$ zeolite), the $\mathrm{CO}_{2}$ adsorption isotherms in powder samples are fully reversible and the gap observed in the argon adsorption isotherms between powder and shaped samples is recovered. Thus, one can conclude from the comparison between argon isotherms at $87 \mathrm{~K}$ and $\mathrm{CO}_{2}$ isotherms at $273 \mathrm{~K}$ that: (i) the binder present in spherical beads of these zeolites does not significantly influence the adsorption mechanism (regardless of the diffusional effects); (ii) the combination of $\mathrm{Ar}$ and $\mathrm{CO}_{2}$ porosimetry allows to quantify the binder amount; and (iii) the presence of different cations according to the considered zeolite does not change the two previous conclusions.

Yet in the case of the $4 \mathrm{~A}$ zeolite (Figure $5 \mathrm{a}$ ), the isotherm of $\mathrm{CO}_{2}$ is reversible in the case of the powder but irreversible in the case of the shaped beads. This fact suggests that the binder used to shape the beads of the $4 \mathrm{~A}$ zeolite strongly interacts with the $\mathrm{CO}_{2}$ molecules. Furthermore, the $\mathrm{CO}_{2}$ uptake is shifted towards higher relative pressure values, indicating that the binders have an impact on the pore filling pressure and on the calculated PSD. Given the inexistence of available NLDFT models for zeolites in the Quantachrome's ASiQwin Software, PSD was obtained by applying a NLDFT model for $\mathrm{CO}_{2}$ adsorption on carbon. Indeed, this model allows performing a qualitative analysis of the effect of the binder. From Figure $5 b$, one can conclude that the binder blocks part of the microporosity and induces an additional pore size, which leads to the observed shift of the filling pressure.

To verify if the irreversibly of $\mathrm{CO}_{2}$ adsorption isotherm on $4 \mathrm{~A}$ zeolite spherical beads samples was due to the pretreatment conditions, several isotherms were recorded with a pretreatment at different temperatures $(523 \mathrm{~K}, 593 \mathrm{~K}$, and $723 \mathrm{~K})$ under secondary vacuum during $12 \mathrm{~h}$. These results are shown in Figure 6a) and confirm that insufficient purification is not the cause of the irreversibility. This latter is also evidenced at a higher temperature (up to $323 \mathrm{~K}$, see Figure 6b). 


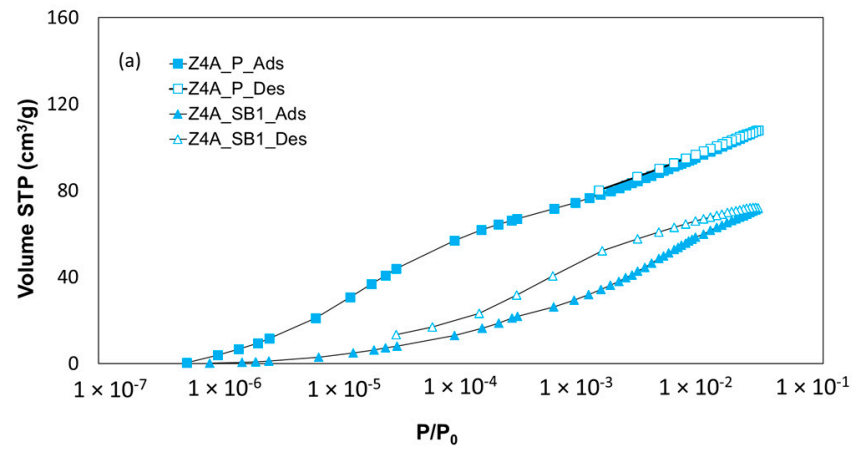

(a)

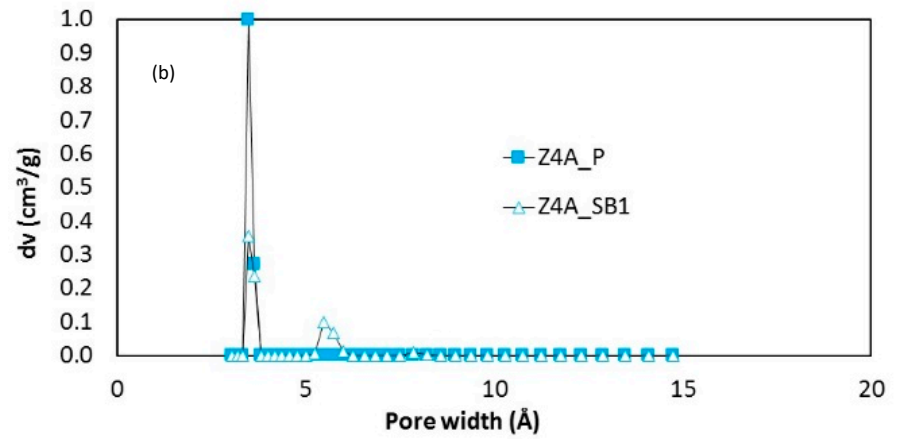

(b)

Figure 5. (a) $\mathrm{CO}_{2}$ adsorption-desorption isotherms at $273 \mathrm{~K}$ on $4 \mathrm{~A}$ zeolite samples, and (b) Pore Size Distribution obtained with a NLDFT model. Adsorption and desorption branches are represented with different symbols in the case of the spherical beads of $4 \mathrm{~A}$ zeolite to highlight the surprising irreversibility of the isotherm.

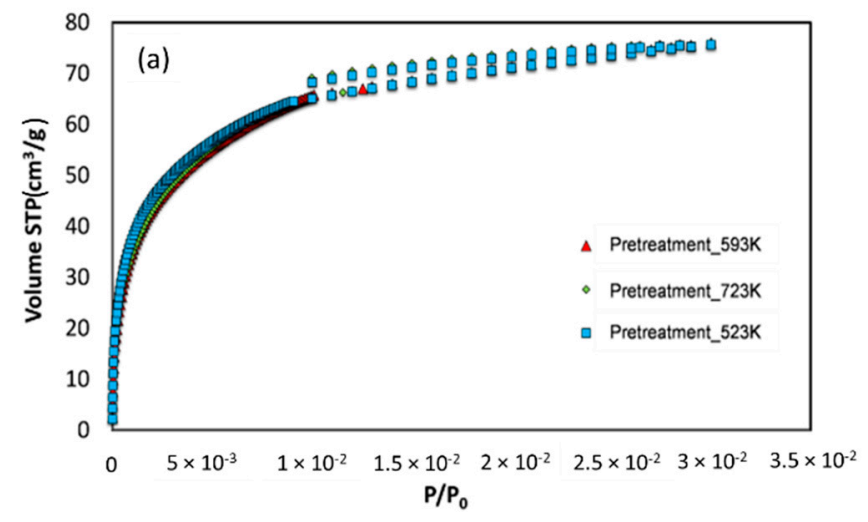

(a)

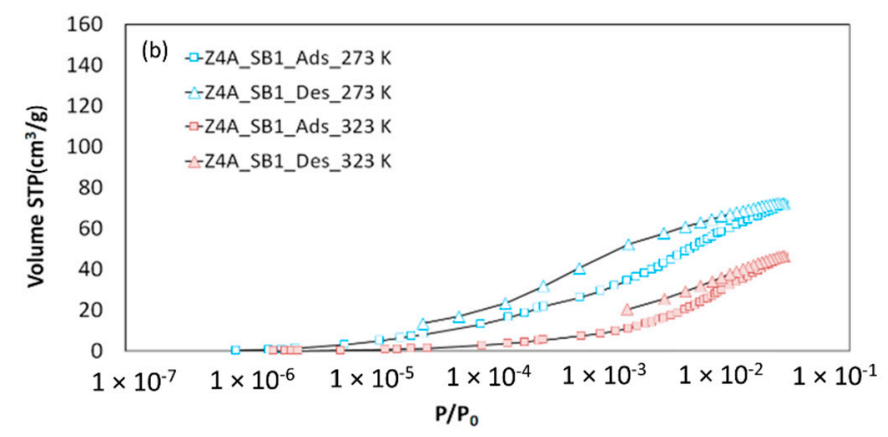

(b)

Figure 6. $\mathrm{CO}_{2}$ adsorption-desorption isotherms on $4 \mathrm{~A}$ zeolite in spherical beads form. (a) At $273 \mathrm{~K}$ after pretreatment at different temperatures, and (b) At $273 \mathrm{~K}$ and $323 \mathrm{~K}$.

\subsection{XRD Analysis}

The obtained diffractograms allow inferring all characteristic peaks for LTA and FAU phases (Figure 7 for 4A zeolite and supplementary information for 13X (Figure S1) and 5A (Figure S2) zeolites).

From the XRD patterns shown in Figure 7, additional small peaks attributed to a $\mathrm{SiO}_{2}$ phase are identified as quartz in spherical bead samples. There are two possible explanations for this occurrence: either there is some $\mathrm{SiO}_{2}$ from the synthesis left inside the zeolite pores or the binder itself contains quartz. In the first case, powder samples should contain this component too, hence the second hypothesis seems more likely. The presence of quartz could also partly contribute to the differences observed between powder and bead-shaped forms of zeolites on $\mathrm{CO}_{2}$ adsorption-desorption isotherms (Figure 1 and Table 2). No other differences are observed from XRD analysis.

\subsection{Infrared Spectroscopy}

The FTIR spectra in the transmission mode of zeolite $4 \mathrm{~A}$ as powder and ground spherical beads are given in Figure 8 and are very similar. The spectra subtraction (ground spherical beads minus powder) allows to evidence the occurrence of quartz in the spherical beads confirming the XRD data. Indeed, the characteristic doublet of quartz at 800 and $780 \mathrm{~cm}^{-1}$ is clearly observed after subtracting the spectra obtained for KBr pellets prepared with a high concentration of the sample (difference spectrum, a) in Figure 8. The difference spectrum obtained with a low concentration of the sample is also compatible with the 
presence of quartz in the spherical beads thanks to the band revealed at $460 \mathrm{~cm}^{-1}$ (b in Figure 8).
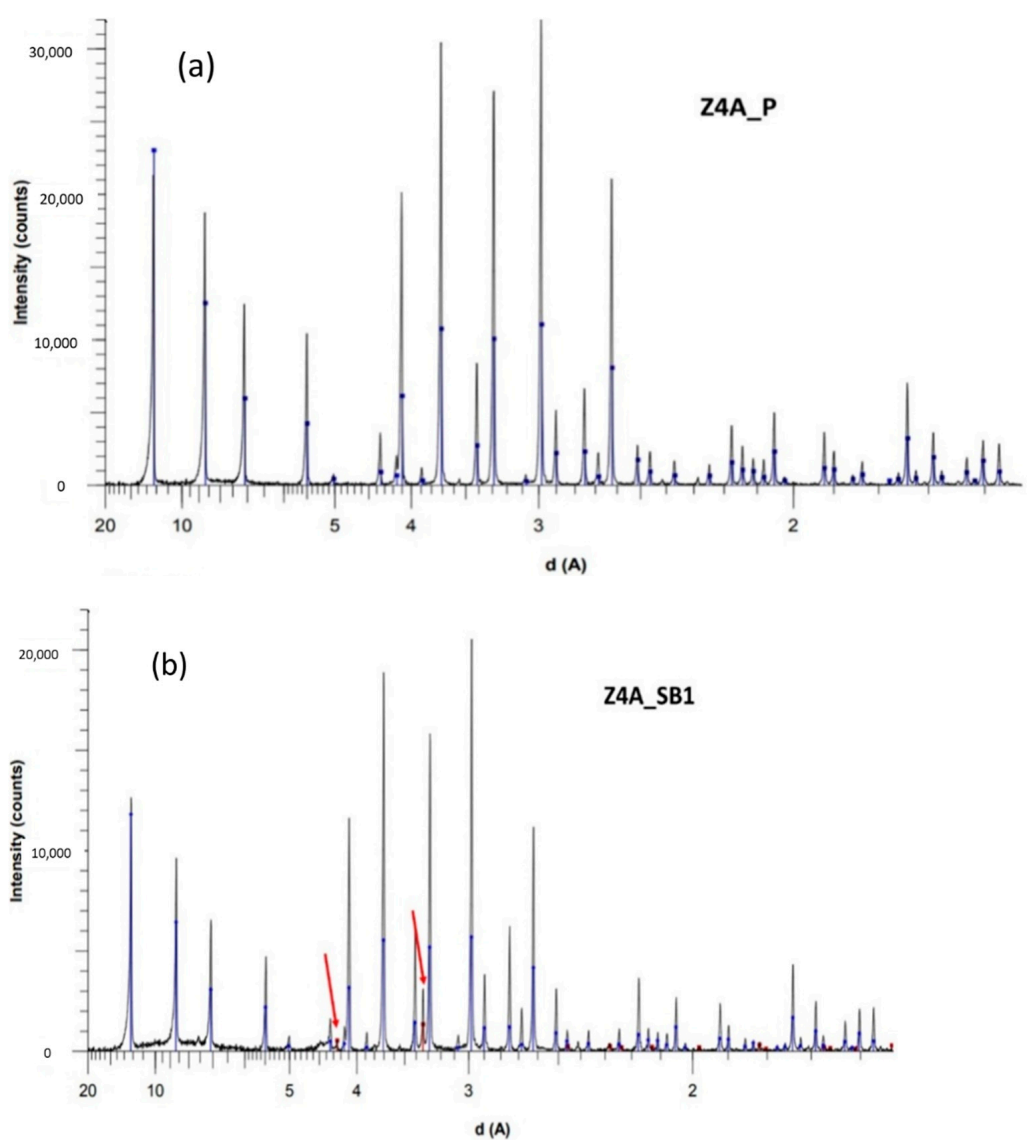

Figure 7. Comparison between $\mathrm{X}$-ray diffractograms on the $4 \mathrm{~A}$ zeolite samples. Experimental patterns of (a) the $4 \mathrm{~A}$ powder samples, and (b) the $4 \mathrm{~A}$ beads samples. Blue lines indicate the theoretical peak position of the ICDD PDF2 data file of the LTA zeolite, red lines indicate the theoretical peak position of the suggested $\mathrm{SiO}_{2}$ phase (highlighted by the arrow symbols).

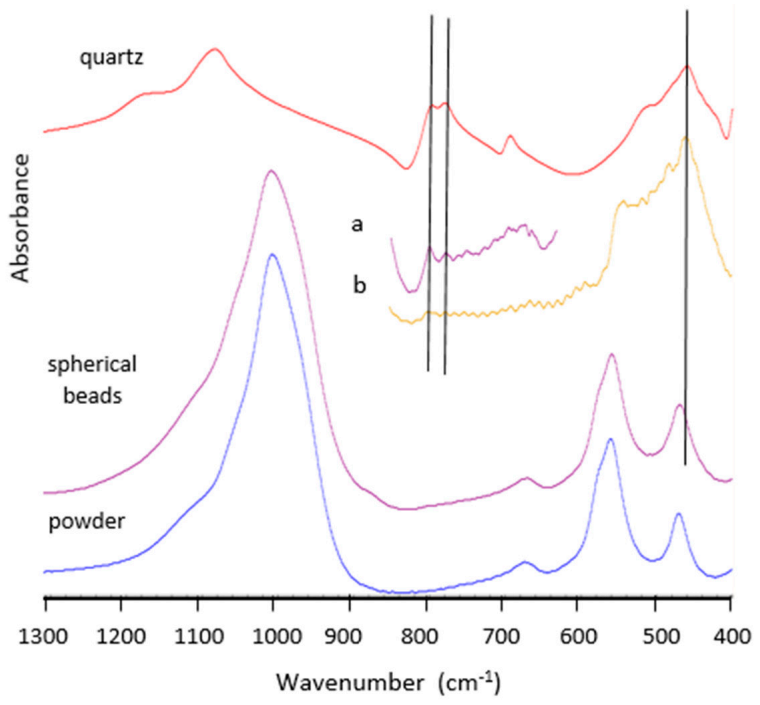

Figure 8. Transmission FTIR spectra of powder and powdered spherical beads for 4A zeolite samples (low concentration samples) and results of spectral subtractions (a) (high concentration samples) and (b) (low concentration samples) and comparison with quartz (HR Minerals library). 
The DRIFT spectra for all zeolite samples are shown in Figure 9. DRIFT spectra reveal a hydrated silicate lattice for all zeolites. For all zeolite samples, a band at $3740 \mathrm{~cm}^{-1}$ (arrow on Figure 9) present for spherical beads and absent for powders indicates the occurrence of protonated sites at the surface of spherical beads.
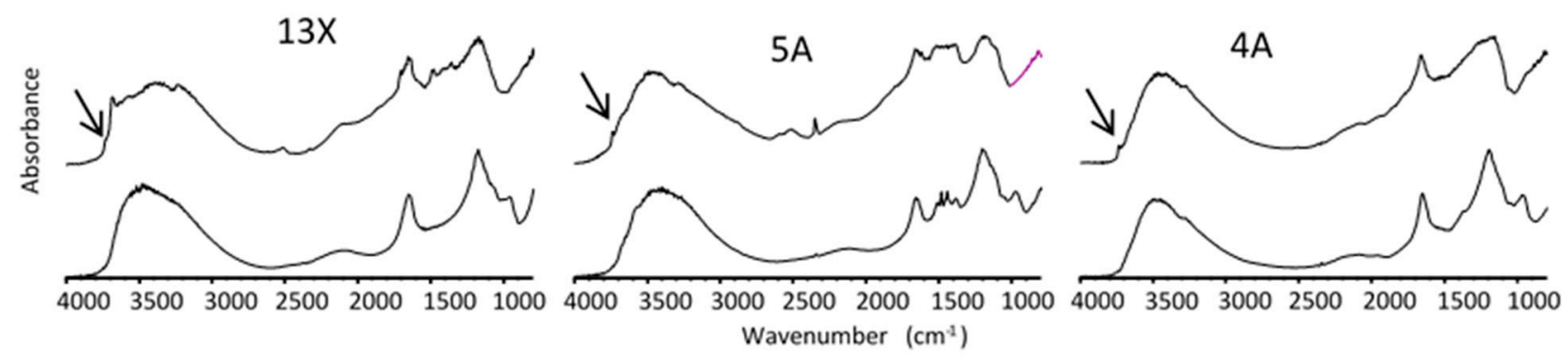

Figure 9. Comparison between DRIFT spectra on different 13X,5A, and 4A zeolite samples as powders (top) or spherical beads (bottom). Arrows indicate the band at $3740 \mathrm{~cm}^{-1}$.

\subsection{ICP-AES Analysis}

The chemical composition of the 4A samples obtained from ICP-AES analysis confirms the results obtained from the other techniques. Indeed, the chemical composition of the powder corresponds to the expected LTA zeolite, with a $\mathrm{Na} / \mathrm{Al}$ ratio close to unity. The beads present substantially higher amounts of both $\mathrm{Si}$ and $\mathrm{Na}$, which can clearly be inferred from the molar Si / Al and $\mathrm{Na} / \mathrm{Al}$ ratios in Table 3. It is further interesting to note that in the powder sample some traces of calcium are present, but even this difference cannot explain the irreversibility of the pelletized $4 \mathrm{~A} \mathrm{CO}_{2}$ adsorption isotherm. These chemical compositions thus further confirm that the extruded samples were not prepared from the zeolite powder as starting material. The higher Si content in the beads compared to the powders are consistent with the detection of quartz in these samples using XRD and DRIFT techniques.

Table 3. ICP-AES analysis results for the 4 A zeolite samples.

\begin{tabular}{|c|c|c|c|c|c|c|c|c|c|c|}
\hline \multirow{2}{*}{ Compound } & \multicolumn{2}{|c|}{ Al $(396.152 \mathrm{~nm})$} & \multicolumn{2}{|c|}{$\mathrm{Na}(396.152 \mathrm{~nm})$} & \multicolumn{2}{|c|}{ Si $(396.152 \mathrm{~nm})$} & \multicolumn{2}{|c|}{ Ca $(396.152 \mathrm{~nm})$} & \multirow{2}{*}{$\begin{array}{c}\text { Molar Si/Al } \\
\text { Ratio }\end{array}$} & \multirow{2}{*}{$\begin{array}{c}\text { Molar } \\
\text { Na/AlRatio }\end{array}$} \\
\hline & $\%$ mass & $\%$ RSD & $\%$ mass & $\%$ RSD & $\%$ mass & $\%$ RSD & $\%$ mass & $\%$ RSD & & \\
\hline Z4A_P & 9.38 & 0.54 & 6.99 & 0.81 & 15.8 & 1.03 & 0.29 & 0.98 & 1.6 & 0.9 \\
\hline Z4A_SB1 & 5.56 & 3.14 & 11.4 & 0.60 & 17.2 & 0.58 & - & - & 3 & 2.4 \\
\hline
\end{tabular}

\section{Conclusions}

A control protocol based on gas porosimetry is proposed to assess the shaping effect on adsorption properties in shaped zeolites. The protocol consists of a combination of argon and $\mathrm{CO}_{2}$ adsorption at $87 \mathrm{~K}$ and $\mathrm{CO}_{2}$ at $273 \mathrm{~K}$ respectively. The comparison of the adsorption of an inert probe molecule such as argon on powdered and shaped samples allows quantifying the amount of binder and its effect on structural properties (pore volume, pore size distribution, and BET surface) for adsorbents with a pore size greater than $4.5 \AA$. The use of a molecule with a smaller kinetic diameter and a quadrupole moment such as $\mathrm{CO}_{2}$ allows determining the effect of the binder in adsorbents with smaller pore size, both on structural properties and adsorption mechanisms of the probe molecule.

The results show that the binder present in both $13 \mathrm{X}$ and $5 \mathrm{~A}$ zeolites does not interact with $\mathrm{CO}_{2}$ and its content is approximately $25 \mathrm{wt} \%$. However, the beads of $4 \mathrm{~A}$ zeolite contain a binder that interacts with $\mathrm{CO}_{2}$ as the probe molecule. This effect is manifested by a shift in the $\mathrm{CO}_{2}$ pore filling relative pressures and the irreversibility of the adsorption isotherm. The irreversibility seems not to be sensitive to the pretreatment temperature (between $523 \mathrm{~K}$ and $723 \mathrm{~K}$ ) nor the operating temperature (between $273 \mathrm{~K}$ and $323 \mathrm{~K}$ ). 
The results have been compared to other experimental techniques such as XRD, IR spectroscopy, and ICP-AES analyses. It was shown that the combination of these classical analytical techniques does not allow to fully identify the nature of the binders (which was not the objective of this work). Further investigations are needed for this purpose. Yet, overall results are very consistent, and the differences observed between the powders and shaped samples validate the control protocol proposed in this work.

Supplementary Materials: The following are available online at https: / www.mdpi.com/article / 10.3390/nano11051205/s1, Figure S1. Comparison between X-ray diffractograms on the 5A zeolite samples. (a) shows the spectra from the 5A powder sample and (b) and (c) show the spectra from the $5 \mathrm{~A}$ beads samples. Orange lines indicate the theoretical peak position of the ICDD PDF2 data file of the LTA zeolite, red lines indicate the theoretical peak position of the suggested $\mathrm{SiO} 2$ phase. Figure S2. Comparison between X-ray diffractograms on the $13 \mathrm{X}$ zeolite samples. (a) shows the spectra from the 13X powder sample and (b) and (c) show the spectra from the 13X beads samples. Magenta lines indicate the theoretical peak position of the ICDD PDF2 data file of the FAU zeolite, black points indicate the theoretical peak position of the suggested $\mathrm{SiO}_{2}$ phase.

Author Contributions: Conceptualization, A.O.-S. and C.M.; methodology, A.O.-S., and C.M.; validation, all the authors; adsorption tests, A.O.-S., F.P., and C.M.; XRD analysis, C.F.; FTIR analysis, E.F., S.P., and J.B.; ICP-AES analysis, A.S.; formal analysis, A.O.-S., C.M., E.F., S.P., C.F. and A.S.; investigation, A.O.-S., and C.M.; writing-original draft preparation, A.O.-S., C.M., S.P., C.F., and A.S.; writing-review and editing, all the authors. All authors have read and agreed to the published version of the manuscript.

Funding: This research was funded by TOTAL EP and ANRT (CIFRE convention $\mathrm{N}^{\circ}$ 2016/1165). A.S., E.F., S.P., and J.B. acknowledge financial support from the European Union (ERDF) and "Région Nouvelle Aquitaine".

Institutional Review Board Statement: Not applicable.

Informed Consent Statement: Not applicable.

Data Availability Statement: Data available on request.

Acknowledgments: C.M. acknowledges E2S UPPA hub Newpores supported by the "Investissements d'Avenir" French program managed by ANR (ANR-16- IDEX-0002). A.S. acknowledges financial support from the European Union (ERDF) and "Région Nouvelle Aquitaine".

Conflicts of Interest: The authors declare no conflict of interest.

\section{References}

1. Gleichmann, K.; Unger, B.; Brandt, A. Industrial Zeolite Molecular Sieves. In Zeolites; Belviso, C., Ed.; IntechOpen: Rijeka, Croatia, 2016.

2. Bingre, R.; Louis, B.; Nguyen, P. An overview on zeolite shaping technology and solutions to overcome diffusion limitations. Catalysts 2018, 8, 163. [CrossRef]

3. Pfenninger, A. Manufacture and use of zeolites for adsorption processes. In Structures and Structure Determination; Baerlocher, C., Bennett, J.M., Depmeier, W., Fitch, A.N., Jobic, H., van Koningsveld, H., Meier, W.M., Pfenninger, A., Terasaki, O., Eds.; Springer: Berlin/Heidelberg, Germany, 1999; pp. 163-198. ISBN 978-3-540-69749-7.

4. Akhtar, F.; Andersson, L.; Ogunwumi, S.; Hedin, N.; Bergström, L. Structuring adsorbents and catalysts by processing of porous powders. J. Eur. Ceram. Soc. 2014, 34, 1643-1666. [CrossRef]

5. Hefti, M.; Marx, D.; Joss, L.; Mazzotti, M. Adsorption equilibrium of binary mixtures of carbon dioxide and nitrogen on zeolites ZSM-5 and 13X. Microporous Mesoporous Mater. 2015, 215, 215-228. [CrossRef]

6. Rioland, G.; Daou, T.J.; Faye, D.; Patarin, J. A new generation of MFI-type zeolite pellets with very high mechanical performance for space decontamination. Microporous Mesoporous Mater. 2015, 221, 167-174. [CrossRef]

7. Lakiss, L.; Gilson, J.P.; Valtchev, V.; Mintova, S.; Vicente, A.; Vimont, A.; Bedard, R.; Abdo, S.; Bricker, J. Zeolites in a good shape: Catalyst forming by extrusion modifies their performances. Microporous Mesoporous Mater. 2020, 299, 110114. [CrossRef]

8. Charkhi, A.; Kazemeini, M.; Ahmadi, S.J.; Ammari Allahyari, S. Effect of bentonite binder on adsorption and cation exchange properties of granulated nano nay zeolite. Adv. Mater. Res. 2011, 335-336, 423-428. [CrossRef]

9. Jasra, R.V.; Tyagi, B.; Badheka, Y.M.; Choudary, V.N.; Bhat, T.S.G. Effect of clay binder on sorption and catalytic properties of zeolite pellets. Ind. Eng. Chem. Res. 2003, 42, 3263-3272. [CrossRef] 
10. Sun, H.; Shen, B.; Liu, J. N-Paraffins adsorption with 5A zeolites: The effect of binder on adsorption equilibria. Sep. Purif. Technol. 2008, 64, 135-139. [CrossRef]

11. Chen, N.-Y.; Liu, M.-C.; Yang, S.-C.; Sheu, H.-S.; Chang, J.-R. Impacts of binder-zeolite interactions on the structure and surface properties of $\mathrm{NaY}-\mathrm{SiO}_{2}$ extrudates. Ind. Eng. Chem. Res. 2015, 54, 8456-8468. [CrossRef]

12. Cao, D.V.; Sircar, S. Heats of adsorption of pure SF6 and CO2 on silicalite pellets with alumina binder. Ind. Eng. Chem. Res. 2001, 40, 156-162. [CrossRef]

13. Shams, K.; Mirmohammadi, S.J. Preparation of 5A zeolite monolith granular extrudates using kaolin: Investigation of the effect of binder on sieving/adsorption properties using a mixture of linear and branched paraffin hydrocarbons. Microporous Mesoporous Mater. 2007, 106, 268-277. [CrossRef]

14. Lowell, S.; Shields, J.E.; Thomas, M.A.; Thommes, M. Characterization of Porous Solids and Powders: Surface Area, Pore Size and Density; Particle, T., Ed.; Springer: Dordrecht, The Netherlands, 2010.

15. Wang, Y.; LeVan, M.D. Adsorption equilibrium of carbon dioxide and water vapor on zeolites 5A and 13X and silica gel: Pure components. J. Chem. Eng. Data 2009, 54, 2839-2844. [CrossRef]

16. Giencke, J. Introduction to EVA. Bruker Coop. Billerica 2007, 31.

17. Chung, F.H. Quantitative interpretation of X-ray diffraction patterns of mixtures. I. Matrix-flushing method for quantitative multicomponent analysis. J. Appl. Crystallogr. 1974, 7, 519-525. [CrossRef]

18. Thommes, M.; Kaneko, K.; Neimark, A.V.; Olivier, J.P.; Rodriguez-Reinoso, F.; Rouquerol, J.; Sing, K.S.W. Physisorption of gases, with special reference to the evaluation of surface area and pore size distribution (IUPAC Technical Report). Pure Appl. Chem. 2015, 87, 1051-1069. [CrossRef]

19. Rouquerol, J.; Llewellyn, P.; Rouquerol, F. Is the BET equation applicable to microporous adsorbents? Stud. Surf. Sci. Catal. 2007, 49-56. [CrossRef]

20. Database of Zeolite Structures. Available online: http:/ /www.iza-structure.org/databases/ (accessed on 31 July 2019 ). 